\title{
RADIOPROTECTION ET RADIOPATHOLOGIE *
}

\author{
M. DELPLA ** \\ (Manuscrit reçu le 2 mai 1968)
}

Les descriptions de cas pathologiques dus aux effets des rayonnements ionisants abondent - surabondent - dans la littérature, non seulement dans les ouvrages et les articles de vulgarisation ou de grande information, mais aussi dans les publications scientifiques. Pour cette raison, avant de plonger dans la radiopathologie, nous allons, si vous le voulez bien, consacrer quelques instants à une OBSERVATION individuelle SPECTACULAIRE... ET RASSURANTE. Ensuite, nous serons plus à l'aise pour étudier l'application des lois de la radiopathologie sur des populations.

Voici l'autohistoradiographie d'une coupe de diaphyse d'un tibia humain (fig. I). Le radium contenu dans le tissu osseux a suffi pour reproduire la forme de l'os sur l'émulsion photographique; en outre, les taches signalent des zones de concentration du radioélément. Le comptage des traces de rayons $\alpha$ a permis de déterminer qu'au niveau de ces taches le débit de dose atteignait $33 \mathrm{rad} / \mathrm{j}$. Parce qu'il s'agit de rayons $\alpha$, auxquels on attribue un facteur de qualité égal à ro, on notera que dans une fraction notable de ce squelette humain le débit d'équivalent de dose dépassait $300 \mathrm{rem} / \mathrm{j}$.

En fait, il s'agit d'une femme qui, contaminée à l'âge de 48 ans dans un but thérapeutique, a vécu jusqu'à 84 ans. Comme durant le traitement par le radium le médecin avait pris soin de prescrire un régime alimentaire carencé en calcium, l'intensité de la fixation a été telle qu'au moment du décès le squelette contenait encore $10,5 \mu \mathrm{Ci}$ de radium 226. Bien que l'observation ne donne pas de précisions, il est possible de retrouver que, durant plus de un an, la malade a absorbé une quarantaine de microcuries de radium par semaine(I). Dans ces conditions, la valeur maximale atteinte par la charge du squelette (environ iso $\mu \mathrm{Ci}$ ) devait donner, dans les zones à concentration élevée, un débit d'équivalent de dose supérieur à $4000 \mathrm{rem} / \mathrm{j}$. Ainsi, en 36 ans, ce squelette a cumulé un équivalent de dose chiffrant: en moyenne, près de 300000 rems; dans les taches, plus de 8000000 de rems.

Et cette femme de longévité enviable n'est même pas morte de cancer ! Toutefois, il serait dangereux de tirer une conclusion générale de l'étude d'un seul cas : sur rog personnes dont la charge de l'organisme en radium 226 dépassait O, I $\mu \mathrm{Ci}$, on a observé I $_{3}$ cancers osseux et ro cancers épithéliaux.

* Allocution du président de la S.F.R.P. pour $1967-68$, prononcée à Saclay, le 26 septembre 1967 , au début de la première réunion scientifique de l'année.

** S G R - E D F, 73 bd Haussmann - 75-Paris (8e).

(I) Activité effectivement passée dans le sang. 
Toute conclusion doit reposer sur de nombreuses observations qui, seules, permettent d'essayer de dégager les LOIS DE LA RADIOPATHOLOGIE, lois qui expriment, pour chacun des effets pathologiques, la relation entre la dose (ou mieux, l'équivalent de dose) et la probabilité d'induction de l'effet considéré.

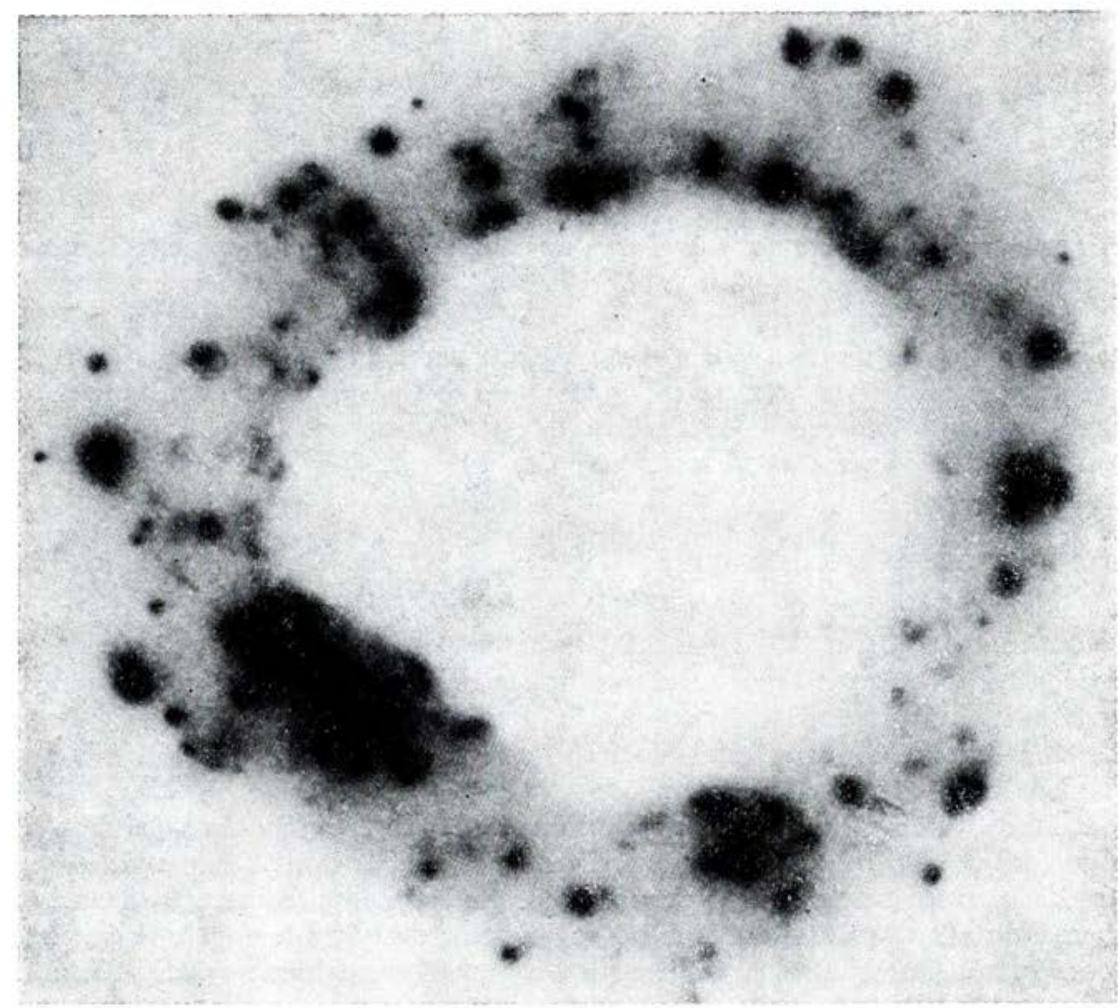

FIG. I. - Auto-historadiographie d'une coupe de tibia d'une personne contaminée par du radium (Rowland, R.E. and Marshall, J.H., Rad. Research II (1959) 30I). Reproduction autorisée.

L'expérimentateur qui désire déterminer l'une de ces lois constitue, par tirage au sort, des groupes de sujets d'effectif suffisant, puis il irradie ces groupes à des niveaux différents; après un délai convenable, il dénombre les sujets qui présentent le symptôme étudié; par commodité, j'appellerai ces derniers les malades. Dans chaque groupe, le rapport du nombre de malades à l'effectif donne un taux; la valeur de ce taux n'est nulle dans aucun des groupes; même pas dans celui qui n'a subi aucune irradiation, où elle correspond au taux spontané. L'expérimentateur peut représenter les résultats en portant en ordonnées, pour chaque valeur d'équivalent de dose, le taux de malades correspondant (fig. 2). La répétition de l'expérience dans des conditions identiques donnerait des points différents : en fait, chaque point n'est déterminé, avec une certaine probabilité (posons-la, pour fixer 
les idées, égale à 0,9$)$ qu'à l'intérieur d'un intervalle de confiance, représenté, sur la figure 2, par un segment de droite parallèle à l'axe des ordonnées. La courbe représentative de la variation du taux de malades en fonction de la dose doit couper la plupart des intervalles de confiance (statistiquement, neuf sux dix). Le tracé, d'autant plus précis que les intervalles de confiance sont plus étroits (c'està-dire les effectifs des groupes plus nombreux) comporte toujours une part d'arbitraire.

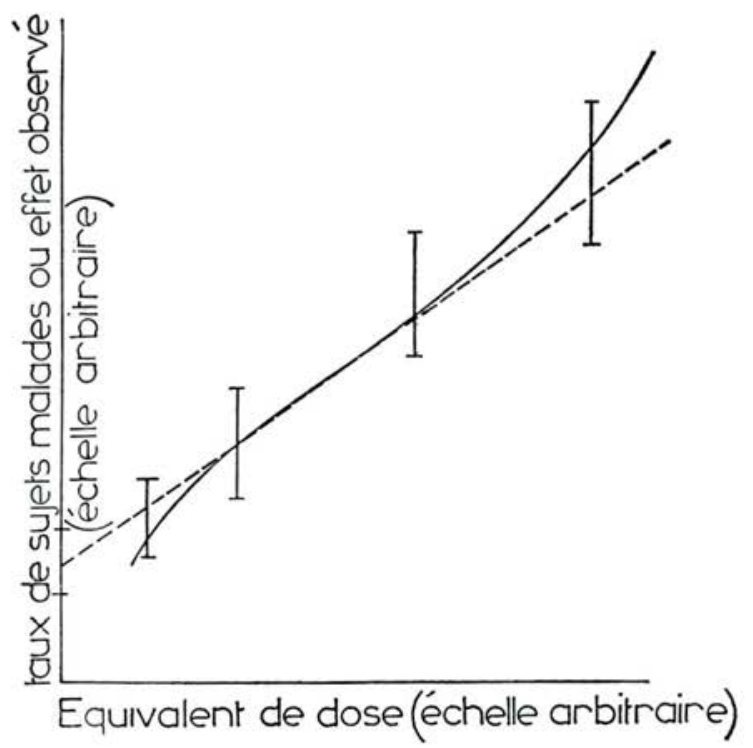

FIG. 2. - Loi de la radiopathologie : représentation graphique de la variation du taux de sujets malades (effet observé) en fonction de l'équivalent de dose.

La courbe la plus simple est, bien sûr, une droite (en tirets, sur la figure 2); la courbe la plus satisfaisante aurait sans doute une allure sigmoïde (en trait continu sur la figure 2).

La lecture des diagrammes est facilitée par une translation de l'axe des abcisses qui place l'origine des coordonnées au point représentatif du taux spontané; ainsi, au lieu de la variation, en fonction de l'équivalent de dose, du taux de malades (effet observé, fig. 2), nous lisons directement la variation du taux de sujets rendus malades (effet induit, fig. 3 ).

La représentation linéaire passant par l'origine implique une relation de proportionnalité entre l'effet induit et l'équivalent de dose. Interprétation fort simple et commode: à toute irradiation, aussi faible soit-elle, correspond une certaine probabilité d'induction de l'effet considéré. Cette probabilité, calculée par simple règle de trois, n'est jamais nulle chez un sujet irradié : il sera reconnu, s'il manifeste cet effet, comme victime de l'irradiation.

La représentation sigmoïde implique l'existence d'un seuil d'action: il n'y a aucun effet induit tant que l'équivalent de dose n'atteint pas la valeur de ce seuil; par 
contre, pour de fortes irradiations, l'existence d'une loi sigmoïde impliquerait l'obtention d'un effet plus important que celui que permet de prévoir la loi de proportionnalité.

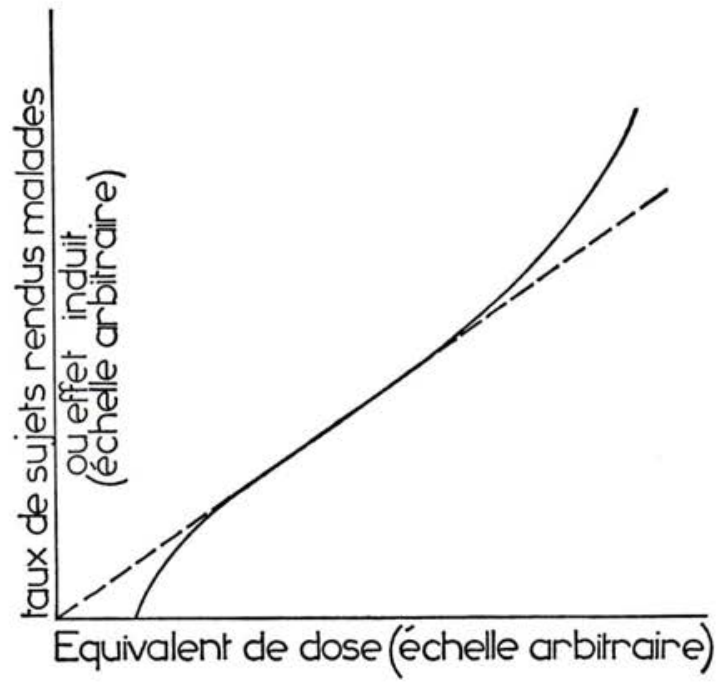

FIG. 3. - Loi de la radiopathologie : représentation graphique de la variation du taux de sujets rendus malades (effet induit) en fonction de l'équivalent de dose.

Quelle que soit leur forme, linéaire ou sigmoïde, les représentations graphiques, confondues sur leur plus grande partie, ne divergent qu'en leurs extrémités. L'étude expérimentale des lois de la radiopathologie doit donc logiquement procéder par étapes : commencer par une étude d'ensemble destinée à donner l'allure du diagramme (essentiellement la pente de sa partie médiane, supposée rectiligne); déterminer ensuite avec autant de précision que possible un certain nombre de points situés aux extrémités.

L'intérêt qui s'attache à préciser la forme des lois de la radiopathologie provient de la gravité des principaux effets des rayonnements ionisants : ils s'appellent cancer et vieillissement prématuré (effets somatiques) ou atteinte de la descendance (effets génétiques); tous, redoutés à juste titre, sont longuement, voire très longuement différés. Ces lois intéressent, non seulement le médecin qui prescrit des irradiations thérapeutiques, ou, beaucoup plus fréquemment, des examens radiologiques, mais encore l'employeur qui expose du personnel en lui faisant utiliser des sources de rayonnements ionisants, et aussi le juriste qui élabore la réglementation, ou en tire des conclusions; enfin, ne l'oublions pas, ces lois intéressent les sujets irradiés eux-mêmes, sujets dont le nombre croît rapidement, et qui, de plus en plus, posent des questions.

Pour répondre à ces questions, dans la multitude des publications scientifiques qui traitent du sujet, je ne retiendrai que les plus connues, sinon les plus valables, et les plus instructives; j'aborderai successivement les effets somatiques, et les effets génétiques. 
Le dénombrement des LEUCÉmIEs (cancer des organes hématopoḯtiques) dans des groupes de malades qui avaient été irradiés sur la colonne vertébrale dans des hôpitaux de Grande-Bretagne, a conduit Court-Brown et Doll, en I957, a admettre, comme bypothèse de travail, une relation de proportionnalité entre la valeur moyenne $x$ de l'exposition mesurée au niveau de la moelle osseuse, exprimée en röntgens, et le taux $y$ de leucémies induites annuellement (taux calculé sur un million d'individus):

$$
y=x .
$$

En 1959, la commission chargée de l'étude des victimes de la bombe atomique (A B C C) a donné, pour Hiroshima, une représentation graphique de la variation du taux de leucémies observées en fonction de la dose. Selon les auteurs, la relation est linéaire dans la gamme des doses qui correspond à des “ données valables ». La pente de la partie rectiligne, égale à 0,8 , diffère peu de la valeur proposée par les auteurs britanniques.

Admettre comme entièrement valable l'hypothèse de travail de ces auteurs conduit à penser qu'un équivalent de dose de I rem augmenterait $y$ de une unité, soit de $2 \%$ le taux annuel de leucémies qui, à cette époque, était en GrandeBretagne, de 49. Plus précisément, Court-Brown et Doll ont déduit de leur hypothèse de travail que pour observer un taux de leucémies statiquement plus élevé que dans le reste de la population, il faudrait dénombrer les leucémies pendant io ans dans un groupe de personnes irradiées d'effectif égal à : 8 ooo pour une exposition à $50 \mathrm{R}$, et à 200000 si l'exposition n'est que de $\mathrm{I} 0 \mathrm{R}$; ou encore : 8 ooo personnes-ans dans le premier cas, 2 millions dans le second.

Leur hypothèse de travail repose sur un total de 40000 hommes-ans, dont seulement $\mathrm{I}_{3} 000$ avaient été exposés à moins de $500 \mathrm{R}$. On ne saurait en attendre un résultat de quelque valeur pour ce qui concerne le domaine des doses faibles. Le diagramme de Hiroshima ne saurait apporter davantage, avec is 000 personnes suivies de 1950 à 1957 (soit seulement durant 8 ans).

Renonçant à suivre 200000 personnes exposées à Io R pendant Io ans (à condition que l'on puisse les trouver) COURT-Brown et DOLL ont orienté leurs recherches dans le domaine des doses faibles sur un groupe de 40000 enfants qui avaient été irradiés in utero à l'occasion de l'examen radiologique du bassin maternel, le fœtus étant présumé beaucoup plus vulnérable que l’adulte. Leurs résultats, publiés en 1960 , contredirent la conclusion à laquelle étaient arrivés STEWART et coll. trois ans auparavant. En 1962, McMahon est venu compléter notre information avec un résultat intermédiaire, fruit d'un énorme travail.

Que penser en présence de ces contradictions? Court-Brown et Doll reconnaissent qu'ils ont considéré un groupe d'effectif un peu faible; de plus, STEWART leur reproche d'avoir arrêté leur enquête à un moment où les enfants, pour la plupart, étaient encore trop jeunes, car, selon elle, les leucémies radioinduites ne se déclarent pas dans les premières années de la vie. McMAHON a fait subir à ses résultats bruts un certain nombre de corrections qui, appliquées à ceux de STEWART, conduisent sensiblement aux mêmes valeurs. Il paraît donc indiqué d'admettre, ce que l'on fait généralement à l'heure actuelle, qu'une exposition globale du fotus humain de l'ordre de un röntgen augmenterait de $40 \%$ environ la probabilité de mourir de cancer, ou de leucémie, dans les dix premières années 
de la vie. En d'autres termes, le fœtus serait bien beaucoup plus sensible que l'adulte pour ce qui concerne l'effet cancérogène des rayonnements ionisants.

SimpSON et coll. avaient conclu dans le même sens, en 1957, à la suite de l'irradiation de tissu lymphoïde hypertrophié (thymus ou végétations adénoïdes) chez des enfants. Il s'agit ici d'une exposition sensiblement plus élevée (en général quelques centaines de röntgens) mais sur une portion de l'organisme étroitement limitée. Ces résultats, complétés récemment (1963) par PifER, Toyooka et coll, sont en contradiction avec ceux de Contr et coll. (1960).

Le groupe d'enfants irradiés observé par Conrr mérite de retenir particulièrement l'attention : is 64 ont reçu iso $\mathrm{R}$ sur le thymus, dans la semaine de leur naissance, non parce qu'ils présentaient une hypertrophie de la glande, mais parce qu'ils sont nés à l'hôpital de Pittsburgh de 1944 à 1946. Sur aucun de ces enfants Conrr n'a trouvé de cancer (il en prévoyait chez eux 2, 5; il en prévoyait et en trouva $s$ dans le groupe témoin, deux fois plus nombreux).

Cette contradiction entre ConTr et SIMPSON a été magistralement interprétée par SAENGER et coll. en I960. Ils ont montré que la différence entre le groupe des enfants irradiés et celui des témoins provient, non de l'irradiation, mais de la constitution des groupes : l'hypertrophie du tissu lymphoïde correspond à un état général déficient où l'on trouve, outre une augmentation de cancers, une augmentation de toutes sortes d'affections; pour beaucoup d'entre elles les rayonnements ne sauraient être incriminés.

N'en va-t-il pas de même chez les enfants d'irradiés in utero? CourT-Brown et Doll le soulignent, mais McMahon l'oublie au moment de conclure : en subdivisant le groupe des enfants irradiés en deux fractions (trouvées sensiblement égales) l'une irradiée par la prise d'un seul cliché, l'autre par plusieurs, on voit que le taux de leucémies ne diffère pas significativement d'une fraction à l'autre. Alors ?

N'est-ce pas que, comme précédemment, la différence entre les enfants du groupe irradié et leurs témoins provient, non de l'irradiation, mais de la sélection des premiers ? Un bassin maternel normal ne fait pas l'objet d'un examen radiologique; il serait surprenant que des êtres qui se sont développés dans un bassin suspect d'anomalie ne diffèrent pas, statistiquement, de ceux qui se sont développés dans un bassin normal.

Pour ce qui concerne la DIMINUTION DE LA LONGÉvitÉ, S. WARREN, en I956, dans une publication de l'académie des sciences des Etats-Unis, dans l'hypothèse d'une relation de proportionnalité, donnait trois points de la courbe représentative de la variation de l'effet induit en fonction de la dose, celle-ci étant repérée de façon seulement très grossière; en effet, il considérait: les médecins radiologistes (très exposés), certains médecins spécialistes (peu exposés) et l'ensemble des autres médecins (supposés non exposés). Selon cet auteur, la longévité des médecins radiologistes (6o,5 ans en moyenne) se trouve raccourcie de 5 ans si on la compare à celle des médecins qui ne font pas de radiologie $(65,6$ ans en moyenne); les médecins peu exposés meurent à un âge intermédiaire.

En I958, SELTZER et SARTwELl, après avoir rappelé que la moyenne arithmétique des âges au décès est une variable inadéquate pour comparer la longévité de deux populations dont la répartition suivant les différentes classes d'âge diffère, ont indiqué que si les médecins radiologistes mourraient comme ceux qui ne font pas de radiologie, leur âge moyen au décès ne serait que de 58,9 ans. 
Le raccourcissement de longévité signalé par WARREN correspond, en réalité, à un allongement de $\mathrm{I}, 6$ an.

La même année, Court-Brown et Doll ont apporté à Genève (à la conférence internationale sur les utilisations pacifiques de l'énergie nucléaire) une note également rassurante. Ils avaient considéré, en Grande-Bretagne : les pionniers de la radiologie, installés avant 1920 (considérablement exposés), les autres médecins radiologistes (exposés) et l'ensemble des médecins (servant de référence). Si cette enquête montra, comme on pouvait s'y attendre, dans le groupe des pionniers, plus de cancers que prévu (essentiellement des cancers de la peau), dans aucun des groupes exposés le nombre de décès n'atteignit le nombre attendu.

Malgré le grand retentissement de la publication de WARREN, je considérais la question comme résolue lorsque, à ma grande surprise, j'entendis cet auteur confirmer son point de vue à la tribune du dernier congrès international de radiologie, à Rome, en septembre 1965 .

Effectivement, Seltzer et SARTwell avaient repris la question en comparant, sur trois périodes différentes (1935-44, 1945-54 et 1955-58) différents groupes de médecins: radiologistes (présumés très exposés), généralistes (dont certains s'exposent) et spécialistes (ophtalmologistes et oto-rhino-laryngologistes, non exposés). Sachant que l'exposition a diminué d'une période à la suivante grâce à l'amélioration de l'appareillage, on dispose de deux groupes exposés à trois niveaux différents. Comme la longévité dans l'espèce humaine s'améliore avec le temps, il fallait un groupe de référence pour chacune des trois périodes.

En considérant des groupes de 1000 sujets, et en leur appliquant, de $s$ en 5 années, de 40 à 80 ans, les taux de mortalité de chacun des groupes de médecins, les auteurs ont trouvé des résultats qui, traduits graphiquement, à titre d'exemple,



FIg. 4. - Variation de l'effectif de groupes de 1000 personnes âgées initialement de 40 ans, auxquels on applique les taux de mortalité des médecins spécialistes ( $\mathrm{S}$ : non exposés aux rayonnements ionisants) généralistes ( $\mathrm{G}$ : parfois exposés) et radiologistes ( $\mathrm{R}$ : exposés). Détermination de l'âge médian et de l'âge moyen au décès (voir les explications dans le texte). 
pour la première période (1935-44), pour les radiologistes (R) les généralistes $(G)$ et les spécialistes (S), sont reportés sur la figure 4. L'âge médian au décès est celui qui correspond à la réduction de moitié de l'effectif. Les deux auteurs se plurent, pour cette période, à retrouver sensiblement le résultat de WARREN : ainsi repérée, la longévité des radiologistes est diminuée de 4,8 ans (de 76,2 à 71,4). Une inégalité se retrouve en toutes périodes et pour toutes les classes d'âge, à l'exception de la plus jeune et pour la dernière période, " ce qui suggère que le danger doit avoir été contrôlé »).

En fait, la considération d'un seul point rend mal compte de la variation d'une grandeur. Pour obtenir un nombre plus représentatif de la façon dont les années qui passent déciment chacun des groupes, pourquoi ne pas considérer la moyenne des ordonnées des points de chacune des courbes de la figure 4 , c'est-à-dire l'âge moyen réel au décès ?

Au milieu de chacun des intervalles (1940, 1950 et 1960) nous avons rapporté (fig. 5), pour chacun des groupes (S, G et R), les valeurs de l'âge médian et de l'âge moyen réel au décès, et joint les points en trait continu pour le premier et en tirets pour le second. Pour la période la plus ancienne, les radiologistes accusent une longévité inférieure seulement de 2,6 ans par rapport à celle des spécialistes non exposés; cette différence, statistiquement très significative, diminue en 1945 - 54 (2, I ans) pour se réduire à 1,4 an en 1954 - 58 (écart de signification incertaine). La longévité augmente dans chacun des trois groupes, et pour les trois, de façon accélérée : tous trois bénéficient de l'amélioration des conditions de vie.

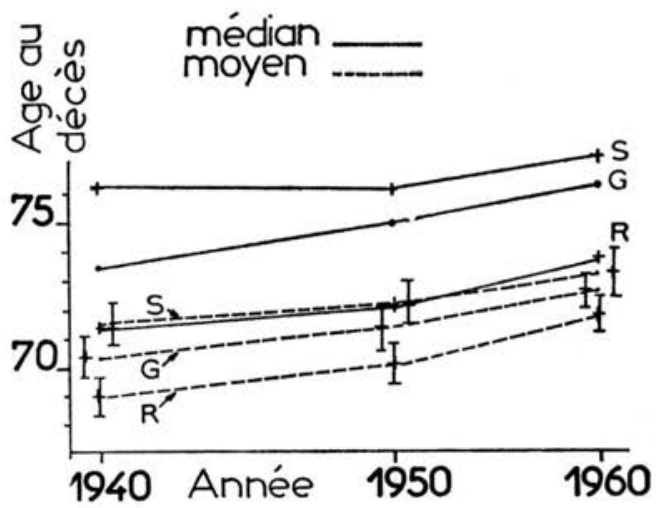

Fig. s. - Variation, dans le temps (1940, r950 et I960), de l'âge médian et de l'âge moyen au décès, pour chacun des groupes considérés : médecins spécialistes ( $\mathrm{S}:$ non exposés aux rayonnements ionisants) généralistes ( $\mathrm{G}$ : parfois exposés) et radiologistes ( $\mathrm{R}$ : exposés).

On voit à quel point la façon de dépouiller les mêmes informations peut influer sur les conclusions. En outre, peut-on attribuer à l'action des rayonnements ionisants les écarts qui subsistent entre les différents groupes de médecins ? Ces écarts ne seraient-ils pas dus, ici aussi, à la sélection ?

SELTzer et SARTwell eux-mêmes soulignent très bien que l'orientation de la carrière d'un médecin vers une spécialité déterminée est orientée, non seulement par ses goûts, mais encore par son état de santé; en particulier certains médecins 
peuvent avoir été intéressés à la radiologie alors qu'en fin de cure sanatoriale ils collaboraient avec leurs confrères, pour lesquels le radiodiagnostic est indispensable. En comparant les médecins radiologistes aux spécialistes pour la période allant de 1935 à 1958 , ils observent que l'excès de mortalité n'est pas dû seulement aux cancers, mais aussi à toutes sortes d'autres causes (rénales et cardiovasculaires en particulier).

Si nous revenons à Hiroshima et Nagasaki, nous voyons que les membres de l'A B C C ont souvent souligné les difficultés de leur tâche et que, en particulier, le diagnostic de cancer ou de leucémie (diagnostic parfois très difficile) est d'autant mieux assuré que le malade se trouvait plus près de la verticale de l'explosion. Des résultats publiés récemment (1965), pris à la lettre, tendraient à attribuer aux personnes des groupes peu irradiés (compris entre I 400 et $2000 \mathrm{~m}$ ) une longévité accrue (I): le taux de mortalité y est moindre que dans le groupe le plus exposé (à moins de $1400 \mathrm{~m}$ ), ce qui se conçoit, mais aussi que dans les groupes les moins exposés (entre 2000 et $2500 \mathrm{~m}$ et au-delà de $2500 \mathrm{~m}$ ).

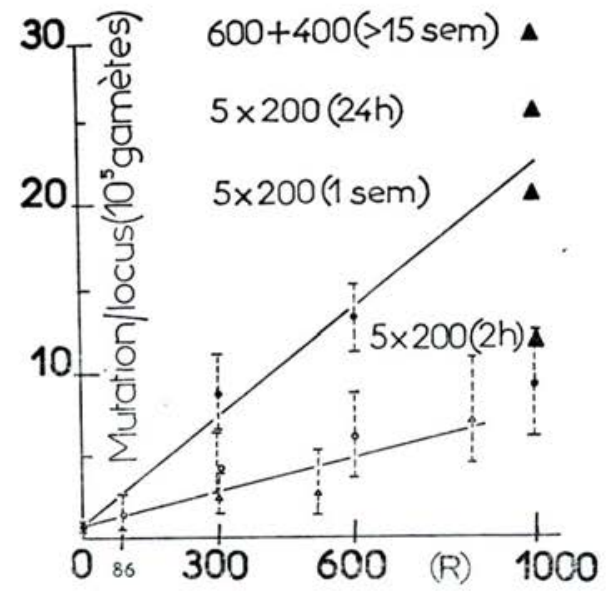

FIG. 6. - Variation du taux de mutations par locus (taux pour roo 000 gamètes) en fonction de l'exposition, suivant les modalités de l'irradiation de souris mâles, d'après les résultats de W.L. Russel et coll., à Oak Ridge. Voir les détails dans le texte.

Puisque nous y sommes, demeurons à Hiroshima pour aborder l'étude des effets génétiques avec la variation du TAUX DE MASCulinité. Schull et NeEL rapportent (1966) qu'aucune variation significative n'a été observée dans la descendance lorsque l'un seulement des parents avait été exposé aux rayonnements de la bombe atomique. Cependant, jusque là, l'ensemble des publications entraînaient la conviction : l'irradiation des gonades du père seul, quatre fois sur six, correspond à une augmentation de ce taux; l'irradiation de la mère seule, cinq fois sur cinq, correspond à sa diminution. Ces auteurs rappellent que l'on ne peut

(1) A ces distances, selon une estimation publiée en 1966, en attribuant aux neutrons un facteur de qualité égal à 2, l'équivalent de dose reçu en un point non abrité situé à la surface du sol aurait été compris entre 65 et 4,5 rems à Hiroshima, entre 200 et 20 rems à Nagasaki. 
retenir le taux de masculinité comme variable, parce qu'il est soumis à de très nombreux facteurs; cela est souvent perdu de vue parce qu'on n'aperçoit que l'élégance de l'argument génétique. Ils espèrent sincèrement n'avoir pas à revenir sur cette question.

Comme pour ce qui concerne les effets somatiques, pour les effets génétiques, l'observation de groupes humains conduit à des conclusions sans valeur. Heureusement la recherche de ces derniers a fait l'objet d'EXPÉrIMENTATIONS VALABLES SUR LA SOURIS.

W.L. RusSEll et coll., à Oak Ridge, à partir d'une lignée de souris homozygotes pour sept caractères récessifs, depuis une quinzaine d'années s'attachent à établir l'allure de la relation entre l'équivalent de dose et l'effet induit. Ils irradient les animaux, soit les mâles, soit les femelles, soit par des rayons $\mathrm{X}$, soit par des rayons $\gamma$, soit par un mélange de rayons $\gamma$ et de neutrons rapides.

L'essentiel de leurs résultats, par irradiation de mâles, est résumé dans la figure 6 où, en fonction de l'exposition des gonades (exprimée en röntgens) on a porté le nombre de mutations observées, rapporté à cent mille souriceaux nouveau-nés (mutation/locus pour $10^{5}$ gamètes). Chaque point est entouré d'un intervalle de confiance dont la grandeur dépend du nombre de souriceaux examinés : très petit pour le taux de mutations spontanées ( 53 I 500 souriceaux) il l'est beaucoup moins pour les autres points (par exemple, seulement 2428 I souriceaux pour $860 \mathrm{R}$ ).

En résumé :

Io Un débit d'exposition de $90 \mathrm{R} / \mathrm{mn}$ (cercles noirs) provoque un taux de mutations trois fois plus élevé que $9 \mathrm{mR} / \mathrm{mn}$ (triangles vides) ou $\mathrm{I} \mathrm{mR} / \mathrm{mn}$ (cercles vides). Ainsi, lorsque le débit d'exposition devient $10^{4}$ fois plus faible, le taux de mutations est divisé par 3 : le débit d'exposition faible permet une restauration partielle, pendant l'irradiation, des désordres qui conduisent aux mutations.

$2^{\circ}$ 1 $000 \mathrm{R}$ à $90 \mathrm{R} / \mathrm{mn}$ en une seule séance (cercle noir) ou en s séances espacées de $2 \mathrm{~h}$ (triangle noir) donnent un taux de mutations situé nettement au-dessous du point attendu sur la droite supérieure. Cela s'explique : après une irradiation aussi élevée, les spermatogonies les plus durement atteintes cessent définitivement de se reproduire, ce sont aussi celles qui ont la plus grande probabilité de porter une mutation. Avec des irradiations fractionnées, tout rentre dans l'ordre : un intervalle de temps suffisant entre les séances permet aux phénomènes de restauration de jouer en assurant la survie des spermatogonies (trois triangles noirs en haut de la figure 6). Par contre, 2 séances de $500 \mathrm{R}$ à $24 \mathrm{~h}$ d'intervalle conduisent à un taux doublé : so (point non représenté sur la figure 6). Ici, la première irradition a sans doute provoqué, après un temps de pause, un certain synchronisme dans les divisions des spermatogonies qui, $24 \mathrm{~h}$ plus tard présentent toutes une sensibilité accrue à l'action mutagène des rayonnements.

$3^{\circ}$ Les taux précédents sont les valeurs moyennes obtenues à partir des 7 locus; or, le taux varie d'un locus à un autre, d'un facteur qui est de l'ordre de 25 entre le plus sensible et le plus stable (valeur à rapprocher des facteurs 3 et 2 trouvés précédemment).

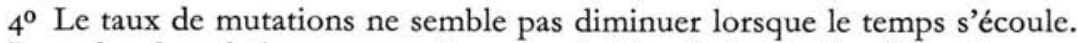

La recherche relative aux conséquences de l'irradiation des femelles est beaucoup moins avancée que la précédente; la figure 7 rapporte l'essentiel des résultats. 
$5^{\circ}$ Les ovocytes se montrent beaucoup plus sensibles que les spermatogonies à la variation du débit d'exposition, ce qui est mis en évidence par le report des droites obtenues précédemment pour les mâles : l'irradiation à $9 \mathrm{mR} / \mathrm{mn}$ (carrés vides) ne paraît pas les affecter; au contraire, avec $90 \mathrm{R} / \mathrm{mn}$, l'exposition à $400 \mathrm{R}$ donne un taux voisin de 20 , contre ro seulement chez le mâle (alors que les spermatogonies ne cessent de se diviser après la puberté, les ovocytes demeurent au repos depuis peu de temps après la naissance jusqu'à peu de temps avant l'ovulation).



FIG. 7. - Variation du taux de mutations par locus (taux pour 100 000 gamètes) en fonction de l'exposition, suivant les modalités de l'irradiation de souris femelles, d'après les résultats de W.L. Russell et coll., à Oak Ridge. Voir les détails dans le texte.

60 Contrairement aux testicules, les ovaires ne conservent pas le souvenir de l'irradiation: le taux de mutations retrouve sa valeur spontanée en quelques semaines.

En résumé, la variation du taux de mutations avec le débit d'exposition montre, contrairement à ce que l'on pensait : un pouvoir de restauration de l'effet mutagène (influence du débit de dose et du fractionnement de la dose), des différences de comportement fondamentales entre les cellules mâles et femelles, une sensibilité très variable d'un gène à un autre.

D'après la façon dont Russell a obtenu sa lignée de souris, on peut penser que les sept gènes observés sont, chez la souris, parmi les plus sensibles.

Considérons l'expérimentation de J.F. SPAlding et coll. qui, à Los Alamos, irradient à $200 \mathrm{R}$ les mâles de chaque génération d'une lignée qu'ils comparent à la lignée non irradiée issue des mêmes parents. A la trentième génération, la lignée irradiée ne paraît pas particulièrement handicapée par rapport à la lignée témoin; cependant, selon les résultats de RuSSELL, si l'on admet 20000 locus par gamète, l'irradiation devrait ajouter, en moyenne, un caractère muté par génération. Effectivement, il semble bien que l'application brutale des résultats de RusSell conduirait à des conclusions par trop pessimistes.

Remarquons par ailleurs que la zone la plus importante, celle des doses faibles, demeure bien peu explorée : un seul point à $86 \mathrm{R}$ avec $\mathrm{I} \mathrm{mR} / \mathrm{mn}$ chez le mâle (fig. 6 ); 
un seul point à so $\mathrm{R}$ et $90 \mathrm{R} / \mathrm{mn}$ chez la femelle (fig. 7). Ce dernier point a conduit les auteurs - qui jusque là admettaient la loi de proportionnalité, donc l'absence de seuil d'action - à écrire : “ Dans l'évaluation du danger génétique pour l'homme, il devrait maintenant apparaitre que le risque dû à de faibles doses d'irradiation à débit élevé doit être plus faible que celui qui avait été calculé sur la base de doses élevées m.

L'ANXIÉté qui pèse sur l'homme au plus profond de son être dès qu'il pense aux sources radioactives et se voit atteint par les rayonnements qu'elles émettent, rend bien difficile l'étude objective de la question que je viens d'esquisser.

Les travaux les plus connus pour établir les lois de la radiopathologie reposent, de façon quasi absolue, sur l'étude de groupes humains. En raison de l'impossibilité de trouver et d'observer suffisamment longtemps des groupes d'effectif suffisant, les résultats comportent une incertitude considérable dans la zone des doses élevées, la plus accessible. Quant à la zone des doses faibles, les travaux qui mettent en évidence un effet pathologique ne résistent pas à la critique qui montre facilement que les effets attribués aux rayonnements ionisants ne sont dus qu'à l'échantillonnage.

Il est heureux que l'expérimentation sur l'animal ait été entreprise à grande échelle pour l'étude des effets génétiques; nous n'avons plus longtemps à attendre pour atteindre le domaine des doses faibles. Pour ce qui concerne les effets somatiques longuement différés, souhaitons que l'expérimentation sur l'animal soit aussi entreprise à grande échelle.

Il faut libérer de leur anxiété, non seulement les travailleurs qui, de plus en plus nombreux, exploitent l'énergie nucléaire, mais encore la plupart des médecins, et aussi le législateur, car un développement excessif des règlements relatifs à la radioprotection, en les rendant difficilement applicables, irait à l'encontre des règles les plus élémentaires de la sécurité; de plus, une réglementation draconienne ne pourrait que contribuer à accroitre cette anxiété. 